\title{
An analysis of total correctness refinement models for partial relation semantics $\mathbf{I}$
}

\author{
MOSHE DEUTSCH, MARTIN C. HENSON, Department of Computer \\ Science, University of Essex, Wivenhoe Park, Colchester, Essex CO4 \\ $3 S Q, U K$ \\ E-mail: \{mdeuts, hensm\}@essex.ac.uk \\ STEVE REEVES, Department of Computer Science, University of \\ Waikato, Private Bag 3105, Hamilton, New Zealand \\ E-mail: stever@cs.waikato.ac.nz
}

\begin{abstract}
This is the first of a series of papers devoted to the thorough investigation of (total correctness) refinement based on an underlying partial relational model. In this paper we restrict attention to operation refinement. We explore four theories of refinement based on an underlying partial relation model for specifications, and we show that they are all equivalent. This, in particular, sheds some light on the relational completion operator (lifted-totalisation) due to Woodcock which underlies data refinement in, for example, the specification language $\mathrm{Z}$. It further leads to two simple alternative models which are also equivalent to the others.
\end{abstract}

Keywords: operation refinement, specification language, specification logic

\section{Introduction}

In this paper we provide a thorough investigation of total correctnessi' operation refinement (that is the degenerate case of data refinement in which simulations are identity functions) where the underlying semantics of specifications is given in terms of partial relations. A major example of such a semantics is the specification language $\mathrm{Z}$, in which a specification denotes a set of bindings which can be construed to be a partial relation between input sub-bindings and output sub-bindings (see [1] $\overline{1}_{1}^{\prime}$, [1] [6] and [is] for accounts of $\mathrm{Z}$ logic and semantics along these lines). Woodcock, in the book [18] (chapter 16), describes a general account of data refinement in which a relational operator (which we will call the Woodcock-completion) plays the crucial underlying role.2. exposition and mathematical account of that relational completion operation by providing a systematic and comprehensive comparison with other possible approaches. In addition, since the theme of combining formal methods is now an important re-

\footnotetext{
$1_{\text {Now that we have established that we are dealing with total correctness, we will drop this qualification: unless we }}$ specify otherwise, we are interested only in total correctness refinement.

2 [1] ( chapter 8) also introduces a similar idea.

L. J. of the IGPL, Vol. 11 No. 3, pp. 285 COxford University Press 2003, all rights reserved
} 
search area, this paper can be seen as contributing both results and methodological techniques which are of use when combining systems with different semantic bases. In order that our general analysis of partial relations can be extended to $\mathrm{Z}$ in particular (which will permit in due course an investigation of the schema calculus) we will base our mathematical investigation on the logic $\mathcal{Z}_{\mathcal{C}}$ described in [11 1$]_{3}^{3}$. This is an extension of higher order logic with schema types and filtered bindings which can be extended conservatively to cover the key features of $\mathrm{Z}$. We will need, in fact, a theory extension of $\mathcal{Z}_{\mathcal{C}}$ which includes additional constants and axioms in order to provide an account of the Woodcock-completion. The base logic and the theory extension, are presented for completeness in appendix in:

Our investigation is essentially proof-theoretic. We have found that the connections and relationships between various theories of refinement can be most clearly demonstrated in this mathematical style. Even where the underlying notions of refinement are model-theoretic, we will nevertheless express them as theories: they are characterised by sets of introduction and elimination rules. This permits a particularly straightforward strategy for demonstrating that theories are equivalent, or related, which is quite general. This strategy is explained in section ${ }_{1}^{4}$ below.

The paper is structured in the following way. We first revise $\mathrm{Z}$ logic, setting some notation and outlining this important application area for the partial relation semantics. We then move on, in section $\underline{3}_{v}$, to provide four theories of refinement, which are prima facie distinct, based on various alternative semantics for specifications: the first is based on sets of implementations, the second on properties of preconditions and postconditions, the third on a relational completion operator, and the last on weakest preconditions. In section are equivalent. We are led to introduce further models in sections motivated by our earlier results. In addition to our conclusions, acknowledgements and references, we conclude with an appendix setting out the mathematical basis for the entire investigation.

\section{Preliminaries}

In this first section we will revise a little $\mathrm{Z}$ logic, settling some notational conventions in the process..$^{\prime \prime}$

In [1] 11, Z schemas, and operation schemas in particular, were formalised as sets of bindings. This captures the informal account to be found in the literature (e.g. [7in] $\left.[1] 8_{1}^{\prime}\right]$ ). In this paper we will use the meta-variable $U$ (with decorations) to range over operation schema. As an example, consider the operation schema:

\footnotetext{
3 Unlike our earlier papers,
$4_{\text {Further and additional detail, including a summary of the ideas introduced here, can be found in appendix }}$ attempts to formalise $\mathrm{Z}$ as it is informally understood. and in 111 . The appendix covers, in addition to a description of the core $\operatorname{logic} \mathcal{Z}_{\mathcal{C}}$ and the theory $\mathcal{Z}_{\mathcal{C}}^{\perp}$, a section demonstrating that $\mathcal{Z}_{\mathcal{C}}^{\perp}$ is conservative over $\mathcal{Z}_{\mathcal{C}}$. Our objective is to make the appendix here sufficiently complete so that it can serve to support the other papers in this series in the future. All the mathematics in the main body of this paper-takes place in the extended theory $\mathcal{Z}_{\mathcal{C}}^{\perp}$ which is described in the appendix. In certain circumstances (e.g. sections $3.2_{1}$ and $\mathbf{B} .4 \mathrm{f}$ below) some theories can be formalised in the core logic $\mathcal{Z}_{\mathcal{C}}$; since this is technically significant, we will indicate the fact explicitly.
} 
$E x_{0}$

$\mathrm{x}, \mathrm{x}^{\prime}: \mathbb{N}$
$\mathrm{x}=0$
$\mathrm{x}^{\prime}<10$

$E x_{0}$ has the the type $\mathbb{P}\left[\mathrm{x}: \mathbb{N}, \mathrm{x}^{\prime}: \mathbb{N}\right]$, and is understood to be a set of bindings of schema type $\left[\mathrm{x}: \mathbb{N}, \mathrm{x}^{\prime}: \mathbb{N}\right]$. Recall that unprimed labels (such as $\mathrm{x}$ ) are understood to be observations of the state before the operation, whereas primed labels (such as $\left.\mathrm{x}^{\prime}\right)$ are observations of the state afterwards. Each operation schema $U$ will have a type of the form $\mathbb{P} T$ where $T$ is a schema type. The type $T$ can, additionally, always be partitioned as the (compatible) union of its input (or before) type $T^{\text {in }}$, and its output (or after) type $T^{\text {out }}{ }^{\prime}$. That is, $T=T^{\text {in }} \curlyvee T^{\text {out }}$ t $^{\prime}$. For the schema $E x_{0}$ we have $T^{\text {in }}=[\mathrm{x}: \mathbb{N}]$ and $T^{\text {out }}=\left[\mathrm{x}^{\prime}: \mathbb{N}\right]$. In this paper, since we are only dealing with operation refinement, we can assume that all operation schemas have the type $\mathbb{P} T$ where $T=T^{\text {in }} \curlyvee T^{\text {out }}$. With this in place we can omit the type superscripts in most places in the sequel.

\section{Definition 2.1 (semantics for atomic schemas)}

$$
[T \mid P]=_{d f}\{z \in T \mid z . P\}
$$

Note that this definition 1 draws bindings from the natural carrier of the type $T$. As a consequence, writing $t(\perp)$ for any binding which projects an instance of the constant $\perp$ (that is: $t . \mathrm{x}=\perp$ for some observation $\mathrm{x}$ ), we have:

\section{Lemma 2.2}

$$
\frac{t(\perp) \in U}{\text { false }}
$$

The bindings $\backslash \mathrm{x} \Rightarrow 0, \mathrm{x}^{\prime} \Rightarrow n \backslash$, where $n<10$, are all elements of $E x_{0}$. In fact there are no other elements in this case. We can introduce the idea of the precondition of the schema (domain of the relation the schema denotes) to express the partiality involved.

Definition 2.3 Let $T^{\text {in }} \preceq V$.

$$
\text { Pre } U x^{V}=_{d f} \exists z \in U \bullet x=_{T^{i n}} z
$$

Proposition 2.4 The following introduction and elimination rules are immediately derivable for preconditions:

$$
\frac{t_{0} \in U \quad t_{0}=T_{T^{i n}} t_{1}}{\operatorname{Pre} U t_{1}} \quad \frac{\operatorname{Pre} U t \quad y \in U, y=T_{\text {in }} t \vdash P}{P}
$$

where $y$ is fresh.

5 In the technical development it is usual to write the operation schema horizontally, with a vertical line separating the declarations from the predicate. 
Clearly, the precondition of $E x_{0}$ is not (and for operation schemas in general, will not be) the whole of $[\mathrm{x}: \mathbb{N}]$ (in general, $T^{\text {in }}$ ). In this sense operation schemas denote partial relations.

Naturally, an immediate question will arise: what does it mean for one operation schema to refine another? More generally, we are asking: what does it mean for one partial relation to refine another? t $^{4}$

\section{A basic analysis of refinement}

We begin by introducing four distinct notions of refinement based on four distinct answers to the questions above and then we go on to compare them. This serves to illuminate them all, particularly the notion based on the Woodcock-completion which is the de facto standard for $\mathrm{Z}$.

\subsection{F-refinement}

To a logician, a specification resembles a theory; so a natural question is: what are the models of the theory? A computer scientist may ask a closely related question: when is a program an implementation of the specification? We will, in this section, consider deterministic programs and model them as (total) functions. We do this, via a standard expedient of introducing a special value $\perp$, which might represent unwelcome behaviour. Such behaviour might be non-termination but it need not, and nothing we shall do with this value commits us to that interpretation. We shall pronounce $\perp$ the abortive value. In [1] 1 in the authors refer to it as undefined which is, we feel, unfortunate in the context of partial relations; we will have much more to say about this below in section 4.4 . In order to introduce this value into the analysis, the technical development below takes place in the extended theory $\mathcal{Z}_{\mathcal{C}}^{\perp}$.

From the logical perspective, we are interested in all the models of a theory, so given a putative model $g$ and a theory $U$, we would be inclined to write:

$$
g \models U
$$

to represent the statement that $g$ is a model of $U$. Within our application area in computer science we might prefer to read this as a relation of implementation. To signal this interpretation we shall in fact write this judgement as:

$$
g \Subset U
$$

to be pronounced " $g$ implements (is an implementation of) $U$ ".

Now an operation schema has been modelled as a partial relation, so we need to consider how an implementation behaves outside the precondition of the schema (domain of the underlying relation). There are degrees of freedom, but we shall, in this paper, permit what we call chaotic models.t' More exactly, we understand silence in the specification to be permission for an implementation to behave in any arbitrary manner,

\footnotetext{
6 If we were not interested in total correctness, we could simply take the subset relation on the partial relations as refinement, and we would then obtain a very well-behaved theory which also makes the schema calculus fully monotonic with respect to refinement but necessarily treats preconditions as triggers (firing conditions).

7 There is an obvious alternative, based on abortive models or what in 110 is effectively the partial model; we will say a little more in section $8_{1}^{1}$ and it will be investigated in our future work.
} 
including the abortive behaviour $\perp$. In other respects we will expect a model (an implementation) to produce a result which is in the relation whenever it is supplied with an input inside the precondition. This leads to the following definition of the modelling (implementing) relation.

\section{Definition 3.1}

$$
g \oplus_{f} U=_{d f}\left(\forall z \in T_{\perp}^{\text {in }} \bullet \operatorname{Pre} U z \Rightarrow z \star(g z)^{\prime} \in U\right) \wedge g \in T_{\perp}^{\text {in }} \rightarrow T_{\perp}^{\text {out }}{ }^{\prime}
$$

Then we can prove the following.

Proposition 3.2 The following introduction and elimination rules are derivable:

$$
\frac{z \in T_{\perp}^{\text {in }}, \text { Pre } U z \vdash z \star(g z)^{\prime} \in U \quad g \in T_{\perp}^{\text {in }} \rightarrow T_{\perp}^{\text {out }}{ }^{\prime}}{g \oplus_{f} U}\left(\epsilon_{f}^{+}\right)
$$

where $z$ is a fresh variable.

$$
\frac{g \oplus_{f} U \quad \operatorname{Pre} U t \quad t \in T_{\perp}^{\text {in }}}{t \star(g t)^{\prime} \in U}\left(\oplus_{f_{\mathrm{o}}}^{-}\right) \quad \frac{g \oplus_{f} U}{g \in T_{\perp}^{\text {in }} \rightarrow T_{\perp}^{\text {out }}}\left(\oplus_{f_{1}}^{-}\right)
$$

This is sufficient technical development to allow us to explore refinement. We can answer the question: when is $U_{0}$ a refinement of $U_{1}$ ? A reasonable answer is: when any implementation of $U_{0}$ is also an implementation of $U_{1}$. After all, we wish to be able to replace any specification $U_{1}$ by its refinement $U_{0}$, and if all potential implementations of the latter are implementations of this former we are quite safe. Thus we are led to:

\section{Definition 3.3}

$$
\widehat{U}={ }_{d f}\left\{z \mid z \oplus_{f} U\right\}
$$

Then we have F-refinement ("F" for function).

\section{Definition 3.4}

$$
U_{0} \sqsupseteq_{f} U_{1}={ }_{d f} \widehat{U_{0}} \subseteq \widehat{U_{1}}
$$

Obvious introduction and elimination rules for F-refinement follow from this definition.

\subsection{S-refinement}

In this section we introduce a purely proof theoretic characterisation of refinement, which is closely connected to refinement as introduced by Spivey in, for example,

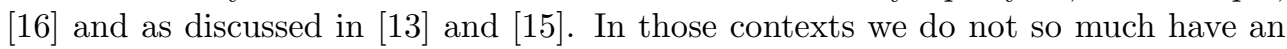
alternative notion of refinement as two sufficient conditions (essentially the premises of the introduction rule in proposition 3.5 below). By adding the two elimination rules we add necessary conditions, and thus formalise an independent theory. There is also a connection with theorem 3.1.2 of [12 20 (page 77), although that analysis concerns 
the two-predicate designs of the refinement calculus (syntactic preconditions) rather than the single predicate specifications of a language like Z (logical preconditions).

This notion is based on two basic observations regarding the properties one expects in a refinement: firstly, that a refinement may involve the reduction of non-determinism, secondly that a refinement may involve the expansion of the domain of definition. Put another way, we have a refinement providing that postconditions do not weaken (we do not permit an increase in non-determinism in a refinement) and that preconditions do not strengthen (we do not permit requirements in the domain of definition to disappear in a refinement).

This notion can be captured by forcing the refinement relation to hold exactly when these conditions apply. S-refinement, named for Mike Spivey, is written $U_{0} \sqsupseteq_{s} U_{1}$ and is given by the definition that leads directly to the following rules:

Proposition 3.5 Let $z, z_{0}, z_{1}$ be fresh variables.

$$
\begin{gathered}
\frac{\text { Pre } U_{1} z \vdash \operatorname{Pre} U_{0} z \quad \operatorname{Pre} U_{1} z_{0}, z_{0} \star z_{1}^{\prime} \in U_{0} \vdash z_{0} \star z_{1}^{\prime} \in U_{1}}{U_{0} \sqsupseteq_{s} U_{1}}\left(コ_{s}^{+}\right) \\
\frac{U_{0} \sqsupseteq_{s} U_{1} \operatorname{Pre} U_{1} t}{\operatorname{Pre} U_{0} t}\left(コ_{s_{0}}^{-}\right) \\
\frac{U_{0} \sqsupseteq_{s} U_{1} \quad \operatorname{Pre} U_{1} t_{0} t_{0} \star t_{1}^{\prime} \in U_{0}}{t_{0} \star t_{1}^{\prime} \in U_{1}}\left(コ_{s_{1}}^{-}\right)
\end{gathered}
$$

This theory does not depend on, and makes no reference to, the $\perp$ value. It can be formalised in the core theory $\mathcal{Z}_{\mathcal{C}}$.

\section{$3.3 W_{\bullet}$-refinement}

Our third notion of refinement is taken directly from the literature [1 $\left.{ }^{-1}\right]$. It is based on a relational completion operator due to Woodcock. For notational convenience we will write $T^{\star}$ for the set $T_{\perp}^{\text {in }} \star T_{\perp}^{\text {out }}$.

The lifted totalisation of a set of bindings (Woodcock-completion) can be defined as follows:

\section{Definition 3.6}

$$
\dot{U}={ }_{d f}\left\{z_{0} \star z_{1}^{\prime} \in T^{\star} \mid \operatorname{Pre} U z_{0} \Rightarrow z_{0} \star z_{1}^{\prime} \in U\right\}
$$

Proposition 3.7 The following introduction and elimination rules are derivable for lifted totalised sets:

$$
\frac{t_{0} \star t_{1}^{\prime} \in T^{\star} \quad \operatorname{Pre} U t_{0} \vdash t_{0} \star t_{1}^{\prime} \in U}{t_{0} \star t_{1}^{\prime} \in \stackrel{\bullet}{U}}\left(\bullet^{+}\right)
$$

and:

$$
\frac{t_{0} \star t_{1}^{\prime} \in \dot{U} \quad \operatorname{Pre} U t_{0}}{t_{0} \star t_{1}^{\prime} \in U}(\bullet-) \quad \frac{t_{0} \star t_{1}^{\prime} \in \dot{U}}{t_{0} \star t_{1}^{\prime} \in T^{\star}}\left(\bullet_{1}^{-}\right)
$$


Note that it is, sometimes, useful to use the following version of $\left(\bullet_{0}^{-}\right)$rule, which is based upon disjunction elimination, rather than implication elimination.

\section{Proposition 3.8}

$$
\frac{t_{0} \star t_{1}^{\prime} \in \dot{U} \quad \neg \operatorname{Pre} U t_{0} \vdash P \quad t_{0} \star t_{1}^{\prime} \in U \vdash P}{P}\left(\bullet \bullet_{\mathrm{o}}^{-}\right)
$$

Lemma 3.9 The following are derivable:

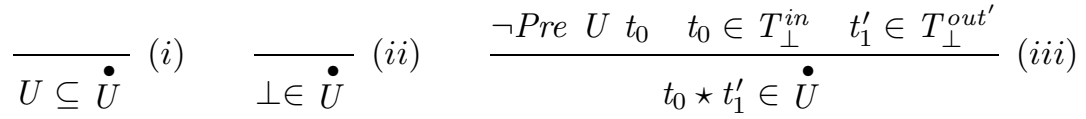

Proof. $(i)$ is trivial. For $(i i)$, consider the following derivation:

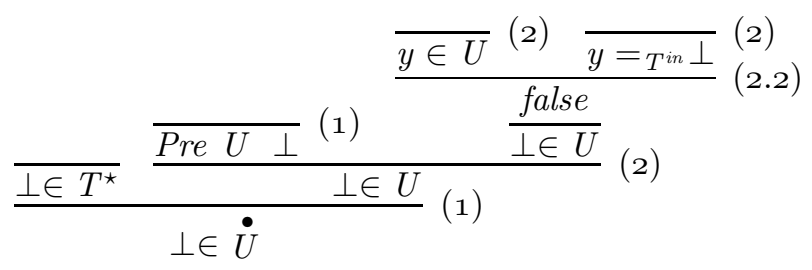

For (iii), consider the following derivation:

$$
\frac{t_{0} \in T_{\perp}^{\text {in }} \quad t_{1}^{\prime} \in T_{\perp}^{\text {out }}}{\frac{t_{0} \star t_{1}^{\prime} \in T^{\star}}{t_{0} \star t_{1}^{\prime} \in \dot{U}}} \frac{\frac{\neg \text { Pre } U t_{0} \overline{\text { Pre } U t_{0}}}{t_{0} \star t_{1}^{\prime} \in U}}{(1)}(1)
$$

Lemmas 3.9 (i), (ii) and (iii) demonstrate that definition 3.6 is consistent with the intentions described in [1 18 ] chapter 16: the underlying partial relation is contained in the completion, the abortive element is present in the relation, and more generally, each value outside the precondition maps to every value in the co-domain of the relation. Furthermore, the following rules, which are derived from lemma 3.9.(iii), embody the non-strict lifting with respect to the abortive element and the fact that everything outside the precondition is mapped onto the abortive value (as well as everything else in the co-domain of the relation).

\section{Corollary 3.10}

$$
\frac{t^{\prime} \in T_{\perp}^{\text {out }}}{\perp \star t^{\prime} \in \dot{U}}(i) \quad \frac{\neg \text { Pre } U t \quad t \in T_{\perp}^{\text {in }}}{t \star \perp^{\prime} \in \dot{U}}(i i)
$$


$W_{\bullet}$-refinement, written $U_{0} \sqsupseteq_{w_{\bullet}} U_{1}$, and named for Jim Woodcock, is defined as follows.

\section{Definition 3.11}

$$
U_{0} \sqsupseteq_{w_{\bullet}} U_{1}=_{d f} \dot{\bullet}_{0} \subseteq \dot{U}_{1}
$$

Obvious introduction and elimination rules follow from this.

\subsection{WP-refinement}

Our final theory of refinement is that based on a weakest-precondition interpretation. In order to formalise this we begin by introducing a notion of postcondition to complement the precondition we introduced earlier.

\section{Definition 3.12}

$$
\text { Post } U z_{0}={ }_{d f}\left\{z_{1}^{\prime} \mid z_{0} \star z_{1}^{\prime} \in U\right\}
$$

Note that this introduces a set, rather than a predicate.

With this in place we can introduce the weakest precondition interpretation of an operation schema. Again, the specified postcondition ( $C$ in the definition below) is expressed as a set rather than as a predicate.

\section{Definition 3.13}

$$
\text { wp } U C=_{d f}\{z \mid \text { Pre } U z \wedge \text { Post } U z \subseteq C\}
$$

The reason why we choose to work with sets, rather than predicates, is simply that it casts the technical material in a similar style to the models we introduced earlier for F-refinement and $\mathrm{W}_{\bullet}$-refinement, which also construct sets from the underlying partial relations.

Proposition 3.14 The following introduction and elimination rules for the weakest precondition of $U$ are derivable:

$$
\frac{\text { Pre } U t \quad z^{\prime} \in \text { Post } U t \vdash z^{\prime} \in C}{t \in w p U C}
$$

where $z$ is a fresh variable.

$$
\frac{t \in w p U C}{\text { Pre } U t} \quad \frac{t_{0} \in w p U C \quad t_{1}^{\prime} \in \text { Post } U t_{0}}{t_{1}^{\prime} \in C}
$$

\footnotetext{
$8_{\mathrm{A}}$ weakest precondition semantics for $\mathrm{Z}$ is providgd in [ [B] , a paper based on the semantics of $\mathrm{Z}$ to be found in the (then) draft $Z$ standard [1] (now superseded by [ $[\boldsymbol{A}]$ ). It would be very interesting to investigate the relationship between the two approaches, but that is beyond the scope of this paper. In passing we note that they provide an interpretation over the syntax of $\mathrm{Z}$ (atomic operation schema expressions) whereas we opt for one over the partial relational semantics (sets of bindings). Generality (an interpretation over all schema expressions) is obtained in two significantly different ways: in ours it follows since all schema expressions denote sets of bindings through the semantics; $[3]$ on the other hand relies on the fact that all schema expressions may be written in the form of an atomic schema. That, in turn, relies on the standard equational logic of schemas. We make some further remarks about our semantic approach, and the equational logic, in section $\mathbf{A}$. These considerations will become even more significant when, in future work, we examine monotonicity issues ${ }^{-}$and what would be necessary to obtain fully monotonic schema calculi for $\mathrm{Z}$.
} 
We can now define WP-refinement.

Definition 3.15

$$
U_{0} \sqsupseteq_{w p} U_{1}={ }_{d f} \forall C^{\mathbb{P} T^{o u t^{\prime}}} \bullet w p \quad U_{1} C \subseteq w p \quad U_{0} C
$$

Proposition 3.16 The following introduction and elimination rules for WP-refinement are derivable:

$$
\frac{z \in w p \quad U_{1} C \vdash z \in w p \quad U_{0} C}{U_{0} \sqsupseteq_{w p} U_{1}}\left(\sqsupseteq_{w p}^{+}\right)
$$

where $z$ and $C$ are fresh variables.

$$
\frac{U_{0} \sqsupseteq_{w p} U_{1} \quad t \in w p \quad U_{1} C}{t \in w p \quad U_{0} C}\left(\sqsupseteq_{w p}^{-}\right)
$$

\section{Lemma 3.17}

$$
\frac{\operatorname{Pre} U t}{t \in w p U(\text { Post } U t)}
$$

Proof.

$$
\frac{\text { Pre } U t \overline{z^{\prime} \in \text { Post } U t}}{(1)}(1)
$$

Since the reverse direction always holds, we have established that $t \in w p U$ (Post $U t$ ) and Pre $U t$ are equivalent.

These interpretations will be reminiscent from accounts such as [1] 197 , although it is unusual to provide a proof-theoretic presentation in terms of introduction and elimination rules. In addition, in [i 14$]$, the preconditions and postconditions of specification statements are syntactic (explicitly given), rather than logical (implicitly given) as they are in Z. Note that our interpretation does not involve the $\perp$ values, and could therefore be formalised in $\mathcal{Z}_{\mathcal{C}}$.

\subsection{Review}

What is the relationship between these four notions of refinement? In particular, can an exploration of that question shed any light on why the Woodcock-completion has been defined in just the way it has? What, in particular, is the role of the value $\perp$ ? Why is the lifting process non-strict with respect to the abortive value? We will begin with the first of these questions.

\section{Four equivalent theories}

In this section we demonstrate that our four theories of refinement are all equivalent. In doing this we will see clearly the critical role that the $\perp$ value plays.

We shall be showing that all judgements of refinement in one theory are contained among the refinements sanctioned by another. Such results will always be established proof-theoretically. Specifically we will show that the refinement relation of a theory 
294 An analysis of total correctness refinement models for partial relation semantics I

$T_{0}$ satisfies the elimination rule (or rules) for refinement of another theory $T_{1}$. Since the elimination rules and introduction rules of a theory enjoy the usual symmetry property, this is sufficient to show that all $T_{0}$-refinements are also $T_{1}$ refinements..$^{1}$

\section{$4.1 \quad$ F-refinement and $W_{\bullet}$-refinement are equivalent $\left(\right.$ in $\left.\mathcal{Z}_{\mathcal{C}}^{\perp}+A C\right)$}

\subsubsection{R-refinement}

We begin this analysis by defining, by way of an intermediate stage, the set of total functions compatible with an operation schema. This forms a bridge between Frefinement and $\mathrm{W}_{\bullet}$-refinement.

\section{Definition 4.1}

$$
\bar{U}={ }_{d f}\left\{z \in T_{\perp}^{\text {in }} \rightarrow T_{\perp}^{\text {out }}{ }^{\prime} \mid z \subseteq \dot{U}\right\}
$$

Then we have:

\section{Definition 4.2}

$$
g \oplus_{r} U=_{d f} g \in \bar{U}
$$

And then R-refinement is simply: $U_{0} \sqsupseteq_{r} U_{1}={ }_{d f} \overline{U_{0}} \subseteq \overline{U_{1}}$ with the usual introduction and elimination rules.

\subsubsection{R-refinement and $\mathrm{W}_{\bullet}$-refinement are equivalent}

We begin by showing that R-refinement satisfies the $\mathrm{W}_{\bullet}$-refinement elimination rule.

Proposition 4.3 The following rule is derivable:

$$
\frac{U_{0} \sqsupseteq_{r} U_{1} \quad t \in \dot{U}_{0}}{t \in \dot{U}_{1}}
$$

Proof. The proof requires the axiom of choice (see the step labelled $(A C)$ below).

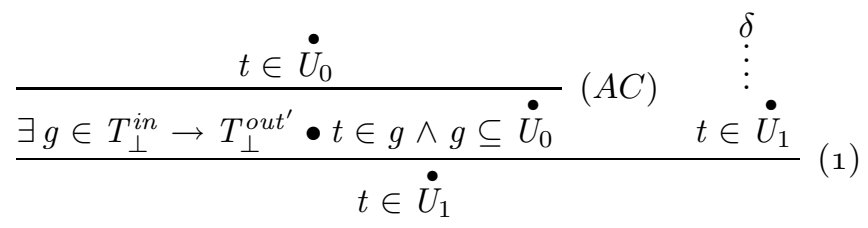

\footnotetext{
9 An alternative strategy would be to show that a similar property holds for the introduction rule. In the refinement theories we consider there is only ever a single introduction rule, and this suggests that this might be a more efficient approach. However, there are as many premises to the introduction rule as there are distinct elimination rules, so in the end the amount of work involved is essentially the, same. Morpover, by considering the elimination rules separately, we can in some cases (see for example sections $\mathbf{A . 2}$ and $\mathbf{4 . 4}$ below) isolate particular properties and reasons underlying equivalence (or non-equivalence in other circümstances) which highlight particular issues of interest.
} 


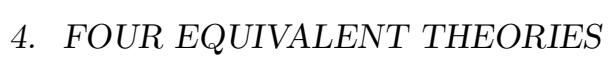

Where $\delta$ is:

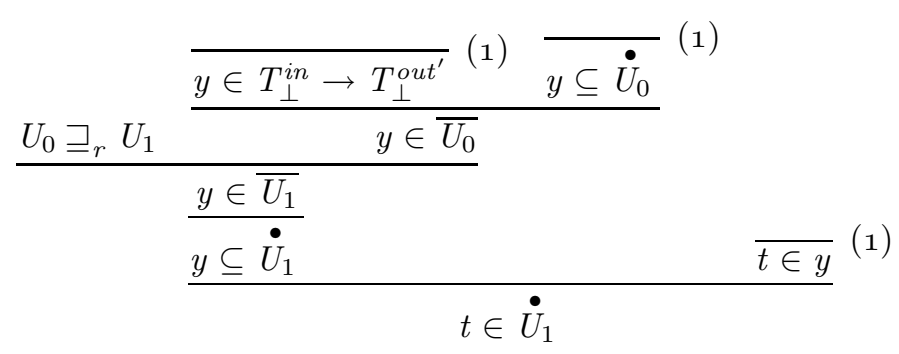

From this we have:

\section{Theorem 4.4}

$$
\frac{U_{0} \sqsupseteq_{r} U_{1}}{U_{0} \sqsupseteq_{w_{\bullet}} U_{1}}
$$

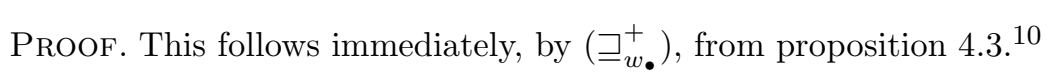

We now show that W.-refinement satisfies the R-refinement elimination rule.

\section{Proposition 4.5}

$$
\frac{U_{0} \sqsupseteq_{w_{\bullet}} U_{1} \quad g \in \overline{U_{0}}}{g \in \overline{U_{1}}}
$$

ProOF.

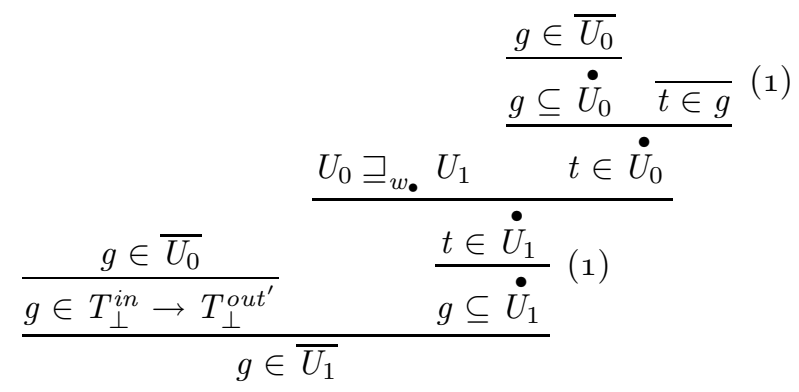

Theorem 4.6

$$
\frac{U_{0} \sqsupseteq_{w_{\bullet}} U_{1}}{U_{0} \sqsupseteq_{r} U_{1}}
$$

Theorems $\overline{4} \overline{4}$ and $\overline{4}$ ingether demonstrate that W.-refinement and R-refinement are equivalent.

\footnotetext{
$10_{\text {The proofs of such theorems are always automatic by the structural symmetry between introduction and elimi- }}$ nation rules. We shall not give them in future.
} 
296 An analysis of total correctness refinement models for partial relation semantics I

\subsubsection{R-refinement and F-refinement are equivalent}

In this case we show that the notions of implementation (rather than refinement) are equivalent by the same strategy involving elimination rules. We first establish that F-implementation implies R-implementation:

Proposition 4.7 The following rules are derivable:

$$
\frac{g \oplus_{f} U}{g \subseteq \dot{U}} \quad \frac{g \oplus_{f} U}{g \in T_{\perp}^{\text {in }} \rightarrow T_{\perp}^{\text {out }}}
$$

Proof.

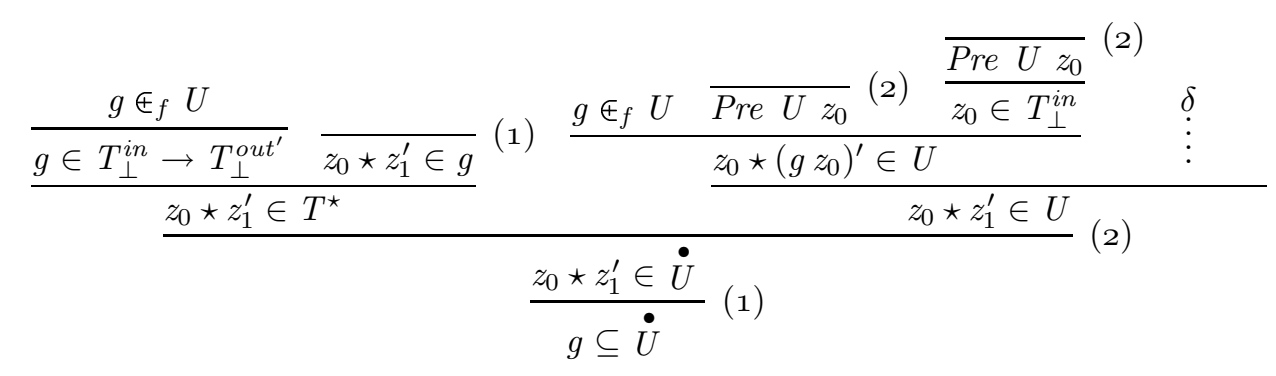

where $\delta$ is:

$$
\frac{\frac{g \oplus_{f} U}{g \in T_{\perp}^{\text {in }} \rightarrow T_{\perp}^{\text {out }}} \overline{z_{0} \star z_{1}^{\prime} \in g}}{z_{1}^{\prime}=\left(g z_{0}\right)^{\prime}}(1)
$$

The second rule is immediate.

\section{Theorem 4.8}

$$
\frac{g \oplus_{f} U}{g \oplus_{r} U}
$$

Now we show that R-implementation implies F-implementation.

\section{Proposition 4.9}

$$
\frac{g \oplus_{r} U \quad \operatorname{Pre} U t \quad t \in T_{\perp}^{\text {in }}}{t \star(g t)^{\prime} \in U} \quad \frac{g \oplus_{r} U}{g \in T_{\perp}^{\text {in }} \rightarrow T_{\perp}^{\text {out }}}
$$

Proof.

$$
\begin{aligned}
& \underline{\frac{g \oplus_{r} U}{g \subseteq \dot{U}} \quad \frac{\frac{g \oplus_{r} U}{g \in T_{\perp}^{\text {in }} \rightarrow T_{\perp}^{\text {out }}}}{t \star(g t)^{\prime} \in g} \quad t \in T_{\perp}^{\text {in }}}
\end{aligned}
$$

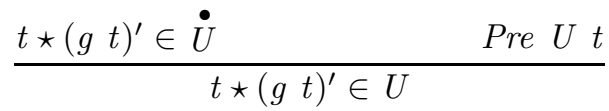

The second rule is immediate. 
Theorem 4.10

$$
\frac{g \oplus_{r} U}{g \oplus_{f} U}
$$

Then, from ' $\overline{4} . \overline{8}$ and $\overline{4} . \overline{1} \overline{1}$, we see that the two notions of implementation are equivalent. Hence, so are the two notions of refinement.

\section{2 $W_{\bullet}$-refinement and S-refinement are equivalent}

We begin by showing that $W_{\bullet}$-refinement satisfies the two S-refinement elimination rules. Firstly the rule for preconditions.

Proposition 4.11 The following rule is derivable:

$$
\frac{U_{0} \sqsupseteq_{w_{\bullet}} U_{1} \quad \text { Pre } U_{1} t}{\text { Pre } U_{0} t}
$$

PRoOF. Consider the following derivation:

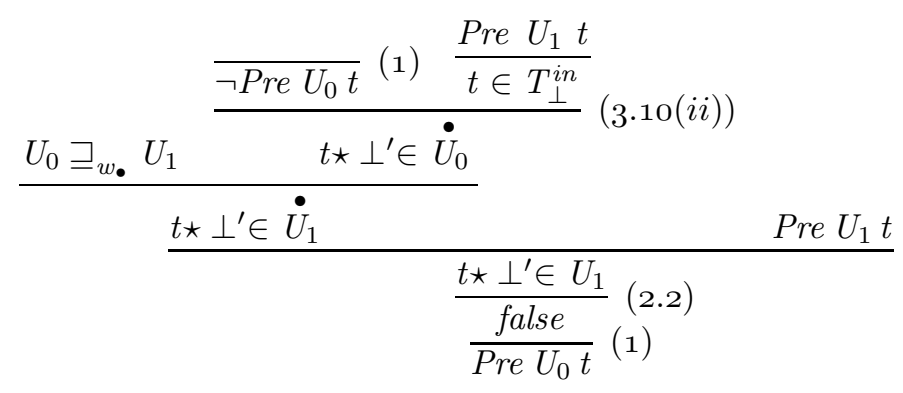

Turning now to the second elimination rule in S-refinement.

Proposition 4.12 The following rule is derivable:

$$
\frac{U_{0} \sqsupseteq_{w_{\bullet}} U_{1} \quad \operatorname{Pre} U_{1} t_{0} \quad t_{0} \star t_{1}^{\prime} \in U_{0}}{t_{0} \star t_{1}^{\prime} \in U_{1}}
$$

Proof.

$$
\frac{U_{0} \sqsupseteq_{w_{\bullet}} U_{1} \quad \frac{t_{0} \star t_{1}^{\prime} \in U_{0}}{t_{0} \star t_{1}^{\prime} \in \dot{U}_{0}}}{\frac{t_{0} \star t_{1}^{\prime} \in \dot{U}_{1}}{\left.t_{0} \star t_{1}^{\prime} \in U_{1}^{\prime}(i)\right)} \quad \text { Pre } U_{1} t_{0}}
$$

Theorem 4.13

$$
\frac{U_{0} \sqsupseteq_{w_{\bullet}} U_{1}}{U_{0} \sqsupseteq_{s} U_{1}}
$$


298An analysis of total correctness refinement models for partial relation semantics $I$

We now show that S-refinement satisfies the $W_{\bullet}$-elimination rule.

Proposition 4.14

$$
\frac{U_{0} \sqsupseteq_{s} U_{1} \quad t_{0} \star t_{1}^{\prime} \in \dot{U}_{0}}{t_{0} \star t_{1}^{\prime} \in \dot{U}_{1}}
$$

ProOF.

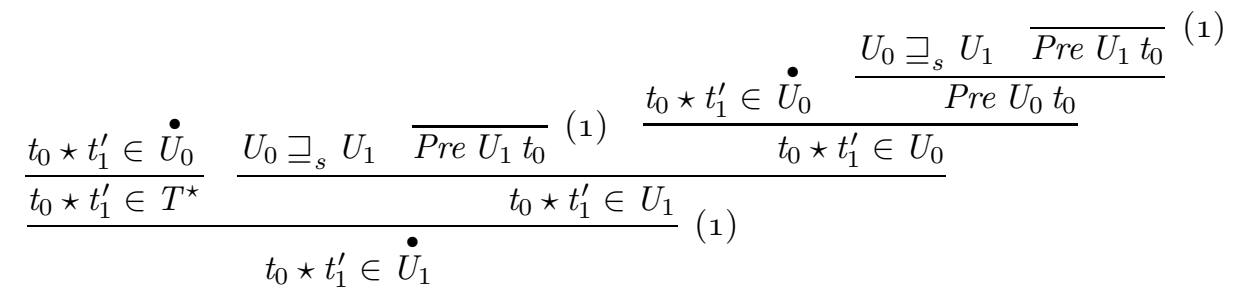

Theorem 4.15

$$
\frac{U_{0} \sqsupseteq_{s} U_{1}}{U_{0} \sqsupseteq_{w_{\bullet}} U_{1}}
$$

Theorems $\bar{A} . \overline{1} \overline{3}$ and $\overline{4} . \overline{1} \overline{5}$ together establish that the theories of S-refinement and $W_{\bullet-}$ refinement are equivalent.

\subsection{WP-refinement and S-refinement are equivalent}

We begin by showing that WP-refinement satisfies the two S-refinement elimination rules. In these results we will often use the fact that $t_{1}^{\prime} \in$ Post $U t_{0}$ and $t_{0} \star t_{1}^{\prime} \in U$ are equivalent without further comment. First we have the rule for preconditions.

\section{Proposition 4.16}

$$
\frac{U_{0} \sqsupseteq_{w p} U_{1} \quad \text { Pre } U_{1} t}{\text { Pre } U_{0} t}
$$

Proof. Consider the following derivation:

$$
\frac{U_{0} \sqsupseteq_{w p} U_{1} \frac{\operatorname{Pre} U_{1} t}{t \in w p U_{1}\left(\text { Post } U_{1} t\right)}}{\frac{t \in w p U_{0}\left(\text { Post } U_{1} t\right)}{\text { Pre } U_{0} t}}
$$

Now the second elimination rule.

Proposition 4.17 The following rule is derivable:

$$
\frac{U_{0} \sqsupseteq_{w p} U_{1} \quad \text { Pre } U_{1} t_{0} \quad t_{0} \star t_{1}^{\prime} \in U_{0}}{t_{0} \star t_{1}^{\prime} \in U_{1}}
$$


Proof. Consider the following derivation:

$$
\begin{aligned}
& \underline{U_{0} \sqsupseteq_{w p} U_{1} \frac{\text { Pre } U_{1} t_{0}}{t_{0} \in w p U_{1}\left(\text { Post } U_{1} t_{0}\right)}(\underline{B} \cdot \overline{1} \overline{1})}
\end{aligned}
$$

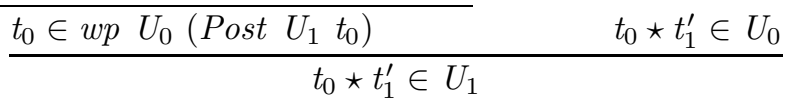

Theorem 4.18

$$
\frac{U_{0} \sqsupseteq_{w p} U_{1}}{U_{0} \sqsupseteq_{s} U_{1}}
$$

We now show that every S-refinement is a WP-refinement.

Proposition 4.19

$$
\frac{U_{0} \sqsupseteq_{s} U_{1} \quad t \in w p U_{1} C}{t \in w p U_{0} C}
$$

Proof. Consider the following derivation:

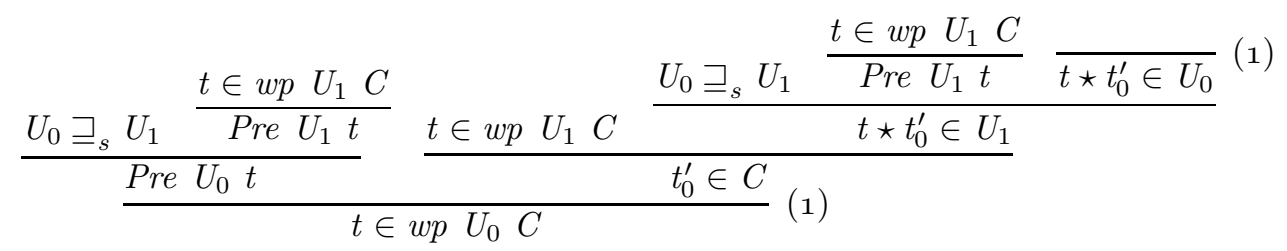

Theorem 4.20

$$
\frac{U_{0} \sqsupseteq_{s} U_{1}}{U_{0} \sqsupseteq_{w p} U_{1}}
$$

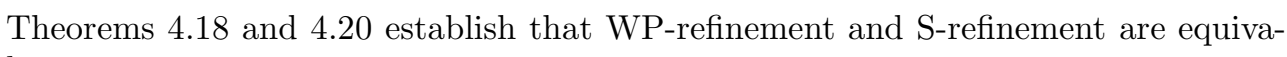
lent.

\subsection{Review}

The model of schemas introduced in W.-refinement not only totalises the schema as a set of bindings, it also introduces the $\perp$ values and extends the domains and codomains accordingly. The totalisation then stipulates chaotic behaviour outside the precondition and additionally for the $\perp$ values.

Why is it necessary to include the new values? What are the consequences of totalisation without lifting?

In [1] [1] the authors explicitly discuss these questions.! consider the particular schema:

$11_{\text {They call the } \perp \text { value "undefined", which is perhaps unfortunate since this is also used with reference to values }}$ outside the domain of definition of particular schemas. We will continue to refer to call it the "abortive" value. 
300 An analysis of total correctness refinement models for partial relation semantics I

$$
\begin{aligned}
& \kappa, \overline{x^{\prime}: \mathbb{N}} \\
& \hline x^{\prime}=0 \\
& \hline
\end{aligned}
$$

This denotes a total constant relation in the model. They then illustrate carefully that, lifting ensures that schema composition is strict with respect to chaotic behaviour.12 On the other hand, totalisation without lifting leads to non-strict recovery from chaos. First we introduce the chaotic specification.

\section{Definition 4.21}

$$
\text { Chaos }=_{d f}[T \mid \text { false }]
$$

Now we describe the non-lifted totalisation, by ensuring that the values are drawn only from natural carrier set rather than the extension including the abortive value.

\section{Definition 4.22}

$$
\stackrel{\diamond}{U}={ }_{d f}\{z \in T \mid \text { Pre } U z \Rightarrow z \in U\}
$$

\section{Proposition 4.23 (Woodcock and Davies)}

$$
\begin{aligned}
& \text { (i) Chaos } \stackrel{\circ}{\circ} \dot{\kappa}={ }_{d f} \text { Chaos } \\
& \text { (ii) Chaos } \stackrel{\circ}{\leftrightarrow} \kappa={ }_{d f} \kappa
\end{aligned}
$$

Proof. See [1]

It should be noted that the second of these results is contingent on the particular choice $\kappa$ : it is not true in general. But this observation notwithstanding, the interpretation of the results requires care. Chaos is described as representing undefinedness, or a run-time error being encountered whatever the initial value. This is odd since, in particular, Chaos permits the input $\perp$ to result in a well-defined output (lifting is non-strict with respect to $\perp$ ). Chaos, as is indicated by our nomenclature, is a relation that permits a chaotic relationship between input and output. It is the relation $\left\{z_{0} \star z_{1}^{\prime} \in T^{\star} \mid z_{1}^{\prime}=\perp^{\prime}\right\}$ that would be closer to what they have in mind (since they refer to $\perp$ as the undefined value). We, for reasons discussed above, will call this Abort.

Now W.-refinement is defined as the subset relation on the Woodcock-completion. Since Chaos is the whole of $T^{\star}$, every schema refines it. In particular the identity relation refines it, and this is the identity for composition. Hence one has: 13

$$
\kappa=\text { Identity }{ }_{9}^{\circ} \kappa \sqsupseteq \text { Chaos }{ }_{9}^{\circ} \kappa
$$

for any $\kappa$, and this would not appear to be recovery from run-time error, but a natural consequence of the general permissiveness inherent in Chaos, indeed, a natural consequence of the fact that the Woodcock-completion is non-strict with respect to the abortive value.

\footnotetext{
12 Note, this is strictness with respect to chaos, not the abortive value.

13 At least when $\kappa$ is e.g. total, and so when composition can be guaranteed to be monotonic. We will explore this further in our companion papers.
} 
Our analysis has, on the other hand, provided a very clear mathematical explanation for lifting: with non-lifted totalisation it is not possible to prove proposition $1.1 \overline{1}$ ' (which requires explicit use of $\perp$ value). Indeed, we can do better: the following is an explicit counterexample.

\section{Definition 4.24}

$$
\text { True }{ }_{d f}[T \mid \text { true }]
$$

Proposition 4.25

$$
\stackrel{\diamond}{\text { True }}=\stackrel{\diamond}{\text { Chaos }}
$$

It is an immediate consequence that the more permissive notion of refinement does not, for example, insist that preconditions do not strengthen.

We have, however, only begun to provide answers to the natural questions that arise. For example, although lifting appears to be necessary, why does it have to be nonstrict with respect to $\perp$ ? Proposition $1 \overline{4} . \overline{2} 5$ ' also raises a question: why is there a distinction between implicit (Chaos) and explicit (True) permission to behave? Note that in the Woodcock-completion, $\stackrel{\bullet}{\bullet}$ rue $\neq$ Chaos .

\section{The non-lifted totalisation}

In the previous section we noted the asymmetry between implicit and explicit chaos. Implicit chaos is more extensive, it permits abortive behaviour that explicit chaos does not allow. This asymmetry seems inevitable in order to obtain a reasonable theory of refinement.!14 This is, as we have shown, indeed the case, unless we re-examine the nature of preconditions.

It seemed only natural to identify the notion of the precondition of a schema with the domain of definition of the underlying relation. There is, however, an alternative approach. Instead of taking a value to be in the precondition when it is related to at least one element of the co-domain of the underlying relation, we could take the condition to be that a value is not related to at least one element of the co-domain of the completed relation. This anticipates the idea that a value which is not in the domain of definition of the underlying relation, will be related to all values in the co-domain after the relation is completed: it excludes from the precondition values in the underlying relation which are already related to all values in the co-domain.

Surprisingly, this leads to a theory which can be formalised entirely in $\mathcal{Z}_{\mathcal{C}}$ (it does not require lifting at all) and which is equivalent to the theories of the previous section. In this way we show that, for operation refinement at least, that lifting of relations is not necessary if one wishes to establish a relational completion semantics for refinement.

\subsection{Preconditions revisited}

In this section we refer to the standard definition of preconditions as $P r e_{0}$ in order to contrast it clearly with the new one.

14 One might take S-refinement to establish minimal conditions. 
$302 \mathrm{An}$ analysis of total correctness refinement models for partial relation semantics I

Definition 5.1 Let $T^{i n} \preceq V$.

$$
\operatorname{Pre}_{1} U z^{V}={ }_{d f} \exists x_{0}^{\prime}, x_{1}^{\prime} \in T^{o u t^{\prime}} \bullet z\left\lceil T^{\text {in }} \star x_{0}^{\prime} \notin U \wedge z \uparrow T^{\text {in }} \star x_{1}^{\prime} \in U\right.
$$

There is a similarity (but not quite an equivalence) here with the total model described in [10] (page 45). His interpretation of specifications as predicates in that model makes use of a similar concept of precondition to Pre $_{1}$, although this is not made explicit.

Proposition 5.2 The following introduction and elimination rules are derivable for preconditions:

$$
\begin{gathered}
\frac{t \star t_{0}^{\prime} \notin U \quad t \star t_{1}^{\prime} \in U \quad t_{0}^{\prime} \in T^{o u t^{\prime}}}{P r e_{1} U t}\left(\text { Pre }_{1}^{+}\right) \\
\frac{\operatorname{Pre}_{1} U t \quad t \uparrow T^{\text {in }} \star y_{0}^{\prime} \notin U, t \uparrow T^{\text {in }} \star y_{1}^{\prime} \in U, y_{0}^{\prime} \in T^{o u t^{\prime}} \vdash P}{P}\left(\text { Pre }_{1}^{-}\right)
\end{gathered}
$$

where $y_{0}$ and $y_{1}$ are fresh variables.

The new notion of preconditions implies the old one.

Lemma 5.3 The following rule is derivable:

$$
\frac{\operatorname{Pre}_{1} U t}{\operatorname{Pre}_{0} U t}
$$

\section{2 $W_{\diamond}$-refinement}

The totalisation (non-lifted) of a set of bindings can be defined as follows:

\section{Definition 5.4}

$$
\stackrel{\stackrel{\leftrightarrow}{U}}{=}{ }_{d f}\left\{z_{0} \star z_{1}^{\prime} \in T \mid \operatorname{Pre}_{1} U z_{0} \Rightarrow z_{0} \star z_{1}^{\prime} \in U\right\}
$$

Proposition 5.5 The following rules are derivable:

$$
\begin{gathered}
\frac{t_{0} \star t_{1}^{\prime} \in T \quad \operatorname{Pre}_{1} U t_{0} \vdash t_{0} \star t_{1}^{\prime} \in U}{t_{0} \star t_{1}^{\prime} \in \stackrel{\diamond}{U}}\left(\diamond^{+}\right) \\
\frac{t_{0} \star t_{1}^{\prime} \in \stackrel{\diamond}{U} \quad \operatorname{Pre}_{1} U t_{0}}{t_{0} \star t_{1}^{\prime} \in U}\left(\diamond_{0}^{-}\right) \quad \frac{t_{0} \star t_{1}^{\prime} \in \stackrel{\diamond}{U}}{t_{0} \star t_{1}^{\prime} \in T}\left(\diamond_{1}^{-}\right)
\end{gathered}
$$

Notice that the values in this completion range over the natural carrier set of the type $\mathrm{T}$.

Lemma 5.6

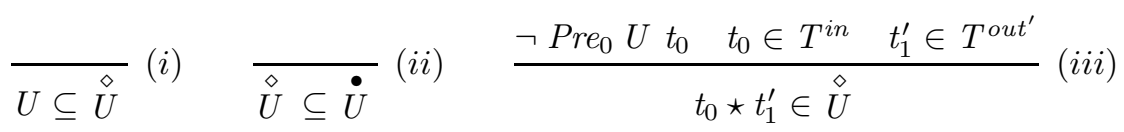


Proof. For $(i i)$, consider the following derivation:

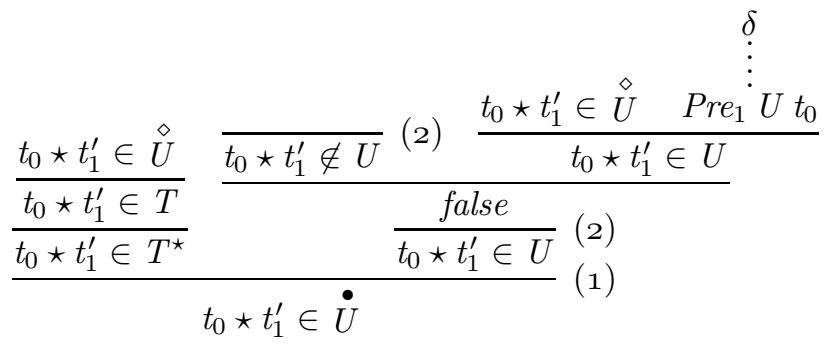

where $\delta$ is:

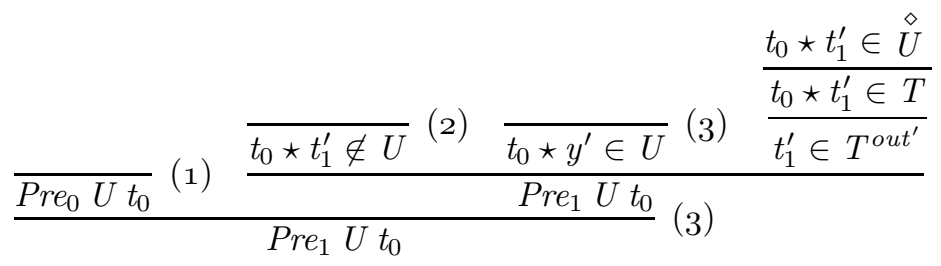

$\mathrm{W}_{\diamond}$-refinement is then defined as follows:

\section{Definition 5.7}

$$
U_{0} \sqsupseteq_{w_{\diamond}} U_{1}={ }_{d f} \stackrel{\diamond}{U}_{0} \subseteq \stackrel{\diamond}{U}_{1}
$$

Obvious introduction and elimination rules are derivable.

\section{3 $W_{\diamond}$-refinement and $S_{1}$-refinement are equivalent}

As we have seen, the abortive value was critical in showing that $W_{\bullet}$-refinement and S-refinement are equivalent. Naturally we should assure ourselves that $\mathrm{W}_{\diamond}$-refinement and a modified version of S-refinement are equivalent.

Let $\mathrm{S}_{1}$-refinement be $\mathrm{S}$-refinement, in which all instances of Pre $_{0}$ are replaced by Pre $_{1}$. We will content ourselves by showing that $\mathrm{W}_{\diamond}$-refinement satisfies the $\mathrm{S}_{1}$-refinement elimination rule concerning preconditions. The remaining elimination rule, and indeed the other direction of the equivalence proof are not significantly different from the proofs we provided earlier in section 4.2 .

Proposition 5.8 The following rule is derivable:

$$
\frac{U_{0} \sqsupseteq_{w_{\diamond}} U_{1} \quad \operatorname{Pre}_{1} U_{1} t}{\operatorname{Pre}_{1} U_{0} t}
$$


$304 A n$ analysis of total correctness refinement models for partial relation semantics I

Proof. Consider the following derivation:

$$
\begin{aligned}
& \begin{array}{c}
\delta \\
\vdots \\
t \star y_{0}^{\prime} \in U_{1} \quad \overline{t \star y_{0}^{\prime} \notin U_{1}}
\end{array} \\
& \frac{\frac{\text { false }}{t \star y_{1}^{\prime} \notin U_{1}} \quad \frac{}{t \star y_{1}^{\prime} \in U_{1}}}{\text { false }}(2) \\
& \frac{\text { Pre }_{1} U_{1} t}{\frac{\text { false }}{\text { Pre }_{1} U_{0} t}(1)}
\end{aligned}
$$

where $\delta$ is:

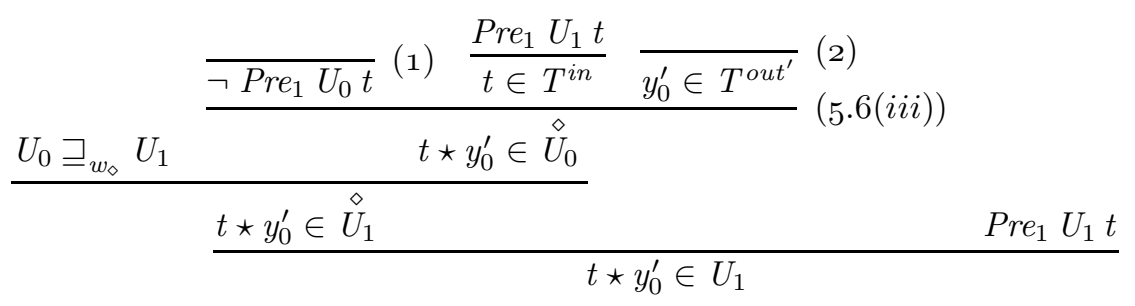

\section{$5.4 W_{\diamond}$-refinement and $W_{\bullet}$-refinement are equivalent (in $\mathcal{Z}_{\mathcal{C}}$ )}

We begin by showing that $\mathrm{W}_{\diamond}$-refinement satisfies the $\mathrm{W}_{\bullet}$-refinement elimination rule, for bindings, which range over the natural carrier set.

Proposition 5.9 Let $t_{0} \star t_{1}^{\prime}$ be a binding with the property that:

$$
t_{0} \star t_{1}^{\prime} \in T
$$

Then the following rule is derivable:

$$
\frac{U_{0} \sqsupseteq_{w_{\diamond}} U_{1} \quad t_{0} \star t_{1}^{\prime} \in \dot{U}_{0}}{t_{0} \star t_{1}^{\prime} \in \dot{U}_{1}}
$$

Proof. Consider the following derivation:

$$
\begin{aligned}
& \underline{t_{0} \star t_{1}^{\prime} \in \dot{U}_{0} \quad \frac{\overline{P r e_{1} U_{0} t_{0}}}{\operatorname{Pre}_{0} U_{0} t_{0}}(1)}\left(5 \cdot 3_{1}^{\prime}\right) \\
& \frac{t_{0} \star t_{1}^{\prime} \in T \quad t_{0} \star t_{1}^{\prime} \in U_{0}}{\diamond}(1) \\
& \frac{U_{0} \sqsupseteq_{w_{\diamond}} U_{1} \quad t_{0} \star t_{1}^{\prime} \in \stackrel{\diamond}{U}_{0}}{\stackrel{\diamond}{U_{1}}} \\
& \frac{t_{0} \star t_{1}^{\prime} \in \dot{U}_{1}}{t_{0} \star t_{1}^{\prime} \in \dot{U}_{1}}\left(\overline{5} \cdot \overrightarrow{\sigma_{1}^{\prime}}(i i)\right)
\end{aligned}
$$


Theorem 5.10 When $\mathrm{W}_{\bullet}$-refinement is understood to range over the natural carriers, then, we have:

$$
\frac{U_{0} \sqsupseteq_{w_{\diamond}} U_{1}}{U_{0} \sqsupseteq_{w_{\bullet}} U_{1}}
$$

Likewise, we show that $\mathrm{W}_{\bullet}$-refinement satisfies the $\mathrm{W}_{\diamond}$-refinement elimination rule.

Proposition 5.11 The following rule is derivable:

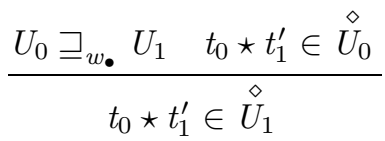

Proof. Consider the following derivation:

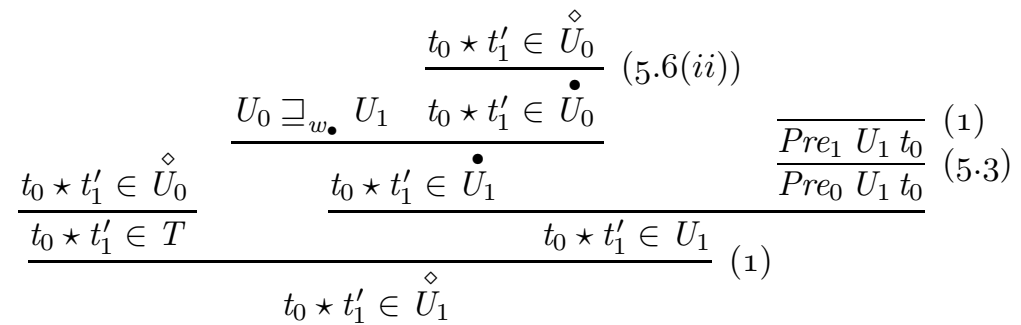

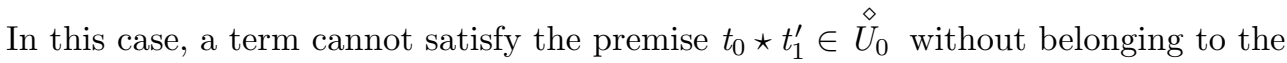
natural carrier, so in this direction no extra condition is necessary.

Theorem 5.12

$$
\frac{U_{0} \sqsupseteq_{w_{\bullet}} U_{1}}{U_{0} \sqsupseteq_{w_{\diamond}} U_{1}}
$$

For terms ranging over the natural carriers, then, the non-lifted and lifted models are equivalent. Since $\mathrm{W}_{\diamond}$-refinement can be formalised in $\mathcal{Z}_{\mathcal{C}}$, and since the carrier sets of $\mathcal{Z}_{\mathcal{C}}$ are the natural carriers of $\mathcal{Z}_{\mathcal{C}}^{\perp}$, we may conclude that we can formalise a form of W-refinement in $\mathcal{Z}_{\mathcal{C}}$ without moving to the extended theory and introducing lifting. The penalty for this is the need for a novel notion of precondition.

\section{The strict-lifted totalisation}

A second question arising from our review was this: why is it necessary to permit recovery from the abortive value in completing the relation? In order to investigate this we consider a final relational completion in which the relation is lifted and totalised, but is strict with respect to abortive behaviour: $\perp$ maps only to $\perp$. 
$306 \mathrm{An}$ analysis of total correctness refinement models for partial relation semantics I

\section{Definition 6.1}

$$
\stackrel{\ominus}{U}={ }_{d f}\left\{z_{0} \star z_{1}^{\prime} \in T^{\star} \mid \text { Pre } U z_{0} \Rightarrow z_{0} \star z_{1}^{\prime} \in U \wedge z_{0}=\perp \Rightarrow z_{1}^{\prime}=\perp^{\prime}\right\}
$$

We obtain obvious introduction and elimination rules, which in this case we will not state explicitly. In addition we have, what are by now, fairly standard properties:

\section{Lemma 6.2}

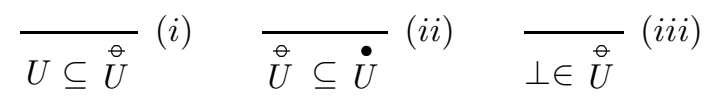

$$
\begin{aligned}
& \frac{\neg \text { Pre } U t \quad t \in T_{\perp}^{\text {in }}}{t \star \perp^{\prime} \in \stackrel{\ominus}{U}}(i v) \quad \frac{\neg \text { Pre } U t_{0} \quad t_{0} \in T^{\text {in }} \quad t_{1}^{\prime} \in T_{\perp}^{\text {out }} \text { ' }^{\prime}}{t_{0} \star t_{1}^{\prime} \in \stackrel{\ominus}{U}}(v)
\end{aligned}
$$

Notice that in $(v) t_{0}$ ranges over the natural carrier set, rather than the extended carrier.

We now introduce $\mathrm{W}_{\ominus}$-refinement.

\section{Definition 6.3}

$$
U_{0} \sqsupseteq_{w_{\ominus}} U_{1}={ }_{d f} \stackrel{\ominus}{U_{0}} \subseteq \stackrel{\ominus}{U_{1}}
$$

Again, we will not state the obvious rules.

\section{$7 \quad \mathrm{~W}_{\ominus}$-refinement and $\mathrm{W}_{\bullet}$-refinement are equivalent}

In the usual manner, we show that $\mathrm{W}_{\ominus}$-refinement satisfies the elimination rule of W.-refinement.

Proposition 7.1 The following rule is derivable:

$$
\frac{U_{0} \sqsupseteq_{w_{\ominus}} U_{1} \quad t_{0} \star t_{1}^{\prime} \in \dot{U}_{0}}{t_{0} \star t_{1}^{\prime} \in \dot{U}_{1}}
$$

Proof. Consider the following derivation:

$$
\begin{aligned}
& \delta_{0}
\end{aligned}
$$

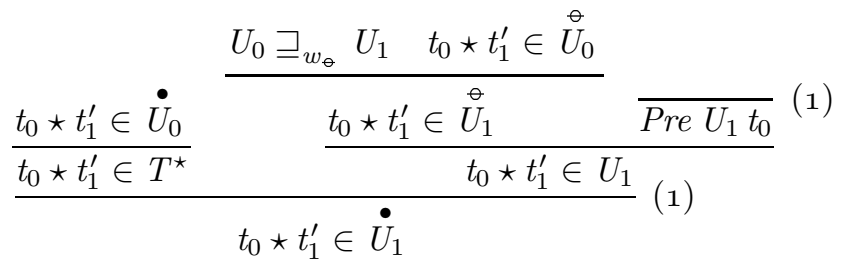


where $\delta_{0}$ is:

$$
\begin{aligned}
& \begin{array}{c}
\delta_{1} \\
\vdots
\end{array} \\
& \underline{U_{0} \sqsupseteq} \underline{w}_{\ominus} U_{1} \quad t_{0} \star \perp^{\prime} \in \stackrel{\ominus}{U_{0}} \\
& \frac{t_{0} \star \perp^{\prime} \in \stackrel{\ominus}{U}_{1} \quad \overline{\text { Pre } U_{1} t_{0}}}{(1)} \\
& \frac{t_{0} \star \perp^{\prime} \in U_{1}}{\text { false }}\left(\overline{t_{0} \star t_{1}^{\prime} \in U_{0}}\right.
\end{aligned}
$$

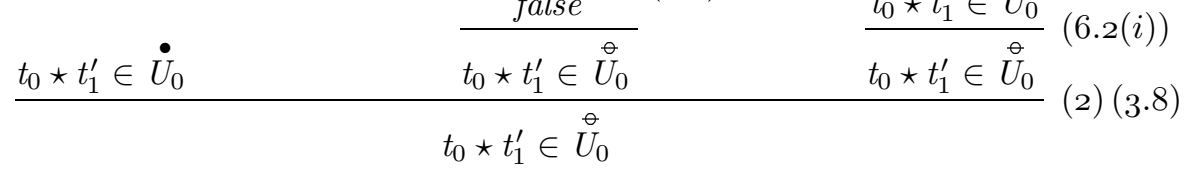

and where $\delta_{1}$ is:

$$
\frac{\frac{t_{0} \star t_{1}^{\prime} \in \dot{U}_{0}}{\operatorname{to}_{0} \star t_{1}^{\prime} \in T^{\star}}}{t_{0} \in T_{\perp}^{i n}}\left(\underset{6 r 2 U_{0} t_{0}}{\operatorname{Pr}}(i v)\right)
$$

Notice the use of the second version of rlue $\left(\bullet \bullet_{0}^{-}\right)$(proposition $\overrightarrow{3} . \overline{8}$ ) in $\delta_{0}$.

Theorem 7.2

$$
\frac{U_{0} \sqsupseteq_{w_{\ominus}} U_{1}}{U_{0} \sqsupseteq_{w_{\bullet}} U_{1}}
$$

Likewise, we prove that $\mathrm{W}_{\bullet}$-refinement implies $\mathrm{W}_{\ominus}$-refinement by proving that $\mathrm{W}_{\bullet}$ refinement satisfies the elimination rule of $\mathrm{W}_{\ominus}$-refinement.

Proposition 7.3 The following rule is derivable:

$$
\frac{U_{0} \sqsupseteq_{w_{\bullet}} U_{1} \quad t_{0} \star t_{1}^{\prime} \in \stackrel{\ominus}{U_{0}}}{t_{0} \star t_{1}^{\prime} \in \stackrel{\ominus}{U_{1}}}
$$

Proof. Consider the following derivation:

$$
\begin{aligned}
& \delta
\end{aligned}
$$

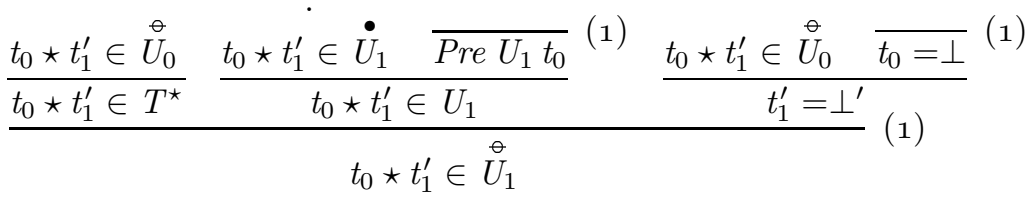


where $\delta$ is:

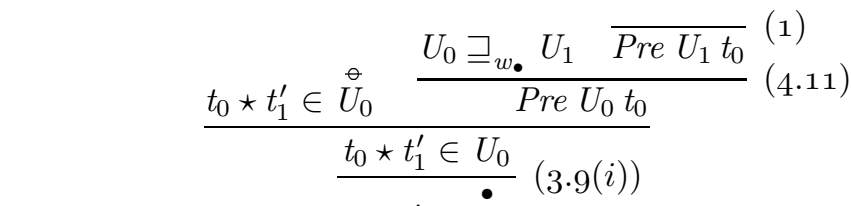

$$
\begin{aligned}
& \frac{U_{0} \sqsupseteq_{w_{\bullet}} U_{1} \quad t_{0} \star t_{1}^{\prime} \in \dot{U}_{0}}{t_{0} \star t_{1}^{\prime} \in \dot{U}_{1}}
\end{aligned}
$$

Theorem 7.4

$$
\frac{U_{0} \sqsupseteq_{w_{\bullet}} U_{1}}{U_{0} \sqsupseteq_{w_{\ominus}} U_{1}}
$$

This concludes the analysis.

\section{Conclusions and future work}

Refinement in the context of partial relational models of specifications is an important sub-area of refinement in general, not least because the standard interpretation of $\mathrm{Z}$ specifications is a partial relational interpretation. There are, as we have demonstrated here, a number of, at least at first sight, distinct notions of refinement in this context. For Z, the standard account is that due to Woodcock and is based on an interesting notion of relational completion which lifts and totalises partial relations. We have shown that this notion is by no means arbitrary, but that there are a number of alternative notions which are equivalent. We have been able to begin to explain some of the mathematical reasons why it is defined in precisely the way it is, particularly why it is necessary to introduce the abortive value $\perp$. Contrary to the argument in [1 18 ."] the critical reason concerns the behaviour of preconditions, rather than the behaviour of refinement under composition. On the other hand a novel notion of what it means for a value to be in the precondition of a specification leads to a Woodcock-like theory in which the relations need not be lifted. There is, in addition, the possibility of insisting that the abortive value is handled in a strict fashion, also leading to an equivalent theory.

It would have been possible to develop the technical material in this paper purely in terms of abstract partial relations, rather than those induced by $\mathrm{Z}$ specifications. Formalisation in $\mathcal{Z}_{\mathcal{C}}$, however, makes dealing with $\mathrm{Z}$ no more complicated than dealing with abstract partial relations, and it has a further advantage which will become clear in the companion papers to follow: we may smoothly extend our analysis to explore refinement in the context of the schema calculus. The specification language $\mathrm{Z}$ has the advantage of permitting large scale specifications to be constructed in a modular and systematic fashion using connectives reminiscent of quantificational logic. The development of $\mathrm{Z}$ logic in, for example, [1] $\left.\overline{1}_{1}\right]$ shows how this algebra of schema expressions can be realised as a calculus by showing how to interpret the connectives and quantifiers as operations on partial relations which have, in general, 
non-equal domains. In order to understand how Z, in particular, handles refinement in the generalised context of the schema calculus, a separate investigation is planned as a sequel to this paper. In particular, monotonicity issues have to be fully explored. It is well-known that the specification forming operations have poor monotonicity properties with respect to refinement in the relational completion semantics, but a thorough investigation, with a careful consideration of all the options, has not to date been undertaken. What we can say already, given the results in this paper, is that there is no likelihood for improved monotonicity properties for $\mathrm{Z}$ if its underlying semantics is to remain based on the partial relation model: every refinement theory we have constructed on this basis is equivalent. It is perhaps worth pointing out that although we only gave an explicit semantics for atomic schemas (see definition 2.1 ) the meta-variables $U$ range over arbitrary sets of bindings of type $T^{\text {in }} \curlyvee T^{\text {out }}$. In other words we can plug in the entire semantics of the schema calculus (in the way described in, for example, [1] affecting the analysis in any way!15. The consequence of this is, then, that all six theories we have described must suffer from the same defects regarding monotonicity properties. Improving upon this position will be one of the themes underlying our further investigation and we will see how the one way forward is to abandon the standard semantics and its equational logic, in favour of an inequational logic of refinement.

There is a well-known alternative to the notion of refinement which we have investigated in this paper. This is the behavioural approach outlined, for example, in "i'i". In this model, preconditions are understood to be firing conditions (or triggers) and may, therefore, not be weakened. A parallel investigation to the one we have described in this paper (with extensions to cover the schema calculus) is possible, and will also be reported in a future paper in this series.

We made comment, in section 3.2 , regarding the relationship between S-refinement and a theorem concerning two-predicate designs appearing in [i $2_{i}^{\prime}$. The semantics of these designs involve a termination value (it is written $\left.o k^{\prime}\right)$. This suggests an interesting generalisation of the work reported here in which two-predicate designs are represented by total relations involving both signals for termination and nontermination. This model will also be reported in future work.

This paper has concentrated on operation refinement, that is the degenerate case of data refinement in which all simulations are identity functions. This was sufficient for our purposes here, but the deeper notion is data refinement, and there are further aspects to the questions we have raised regarding the Woodcock-completion which do not emerge until data refinement is considered. This is why we emphasised the word "begin" earlier in this concluding section. Operation refinement is sufficiently interesting mathematically to motivate the range of possibilities we have explored here, but there are divergences and surprises to uncover when non-trivial simulations are permitted. This topic will also be covered in a companion paper, making links and comparison between our proof-theoretic approach and the largely semantic approach generally taken in

Our analysis of weakest precondition semantics is here restricted to the atomic schema

$15_{\text {However, one can reasonably argue that, by insulating the schema logic (based on the partial relation interpre- }}$ tation) from the refinement logic (based on any one of the six theories we have described) $\mathrm{Z}$ effectively reduces its refinement logic to the atomic schema case. This is because the schema logic permits the derivation of an equational logic which makes all schema expressions equal to an atomic schema. 
case, and to operation refinement. Some interesting work, developing one or other of these dimensions, has been undertaken, for example by Woodcock and Cavalcanti [2] [ind by Groves setting, of an extension to what we have shown in this paper and with our prooftheoretic methodology, is both simple and revealing. It will form an additional phase of our exploration of refinement and we plan to cover the topic in another paper in this series.

\section{Acknowledgements}

We would like to thank the EPSRC (grant reference: GR/L57913), the Royal Society of Great Britain and the New Zealand Foundation for Research, Science and Technology (grant reference: UOWX0011) for financially supporting this research. Moshe Deutsch is supported by the British Council through an ORS award.

This work has been influenced in its development by too many people to name explicitly. However, special thanks for particularly important discussions and comments go to Rob Arthan, Lindsay Groves, Greg Reeve, Ray Turner and Jim Woodcock.

\section{References}

[1] S. Brien and J. Nicholls. Z base standard version 1.0. Technical report, University of Oxford, 1992.

[2] A. Cavalcanti. A Refinement Calculus for Z. PhD thesis, University of Oxford, 1997.

[3] A. Cavalcanti and J. C. P. Woodcock. A weakest precondition semantics for Z. The Computer Journal, 41(1):1-15, 1998.

[4] A. Cavalcanti and J. C. P. Woodcock. ZRC - a refinement calculus for Z. Formal Aspects of Computing, 10(3):267-289, 1998.

[5] W. P. de Roever and K. Engelhardt. Data refinement: model-oriented proof methods and their comparison. Prentice Hall International, 1998.

[6] J. Derrick and E. Boiten. Refinement in $Z$ and Object-Z: Foundations and Advanced Applications. Formal Approaches to Computing and Information Technology - FACIT. Springer, May 2001.

[7] A. Diller. Z: An introduction to formal methods. J. Wiley and Sons, $2^{\text {nd }}$ edition, 1994.

[8] I. Toyn (ed.). Z Notation: Final committee draft, CD 13568.2. Z Standards Panel, 1999. ftp://ftp.york.ac.uk/hise_reports/cadiz/ZSTAN/fcd.ps.gz.

[9] L. Groves. Evolutionary software development in the refinement calculus. PhD thesis, Victoria University, 2000.

[10] J. Grundy. A method of program refinement. PhD thesis, University of Cambridge, 1993.

[11] M. C. Henson and S. Reeves. Investigating Z. Logic and Computation, 10(1):43-73, 2000.

[12] C.A.R Hoare and J. He. Unifying Theories of Programming. Prentice Hall International, 1998.

[13] S. King. Z and the Refinement Calculus. In D. Bjørner, C. A. R. Hoare, and H. Langmaack, editors, VDM '90 VDM and $Z$ - Formal Methods in Software Development, volume 428 of Lecture Notes in Computer Science, pages 164-188. Springer-Verlag, April 1990.

[14] C. C. Morgan. Programming from Specifications. Prentice Hall International, $2^{\text {nd }}$ edition, 1994.

[15] B. Potter, J. Sinclair, and D. Till. An introduction to formal specification and Z. Prentice Hall, $2^{\text {nd }}$ edition, 1996.

[16] J. M. Spivey. The $Z$ notation: A reference manual. Prentice Hall, $2^{\text {nd }}$ edition, 1992.

[17] J. C. P. Woodcock and S. Brien. $\mathcal{W}$ : A logic for Z. In Proceedings of ZUM'91, 6th Conf. on Z. Springer Verlag, 1992.

[18] J. C. P. Woodcock and J. Davies. Using Z: Specification, Refinement and Proof. Prentice Hall, 1996. 


\section{A The specification logic $\mathcal{Z}_{\mathcal{C}}$ and the theory $\mathcal{Z}_{\mathcal{C}}^{\perp}$}

\section{A.1 $\mathcal{Z}_{\mathcal{C}}$}

\section{A.1.1 The language of $\mathcal{Z}_{\mathcal{C}}$}

$\mathcal{Z}_{\mathcal{C}}$ is an extension of higher order logic with schema types. We begin with the types:

$$
T::=\mathbb{B}|\mathbb{N}| \mathbb{P} T|T \times T|\left[\cdots \mathbf{z}^{T} \cdots\right]
$$

We will often permit the meta-variable $D$ to range over sequences of labels such as $\cdots \mathbf{z}_{i}^{T_{i}} \cdots$ (the order is not important), also writing $\alpha[D]$, when $D$ is $\cdots \mathbf{z}_{i}^{T_{i}} \cdots$, for the alphabet set (in the metalanguage) of labels $\left\{\cdots z_{i} \cdots\right\}$. No label may occur more than once in such a type.

Types of the form $[D]$ are called schema types. The symbols $\preceq, \curlywedge, \curlyvee$ and - denote the schema subtype relation, and the operations of schema type intersection, (compatible) schema type union and schema type subtraction.

All categories of the language of $\mathcal{Z}_{\mathcal{C}}$ must be well-formed with respect to these types.

Next we have the category of terms: We assume the existence of a denumerable set of variables for each type $T$. We use $t$ as a meta-variable over terms of arbitrary type.

The syntax of terms is then:

$$
\begin{array}{ll}
t^{T} & :=x^{T}\left|t^{\left[\cdots \mathbf{z}^{T} \cdots\right]} . l\right| t^{T \times T_{1}} .1 \mid t^{T_{0} \times T} .2 \\
t^{[D]} & ::=t^{T_{2}} \mid[D] \\
t^{\left[\cdots \mathbf{z}^{\mathrm{T}} \cdots\right]} & \left.::=\emptyset \cdots \mathbf{z} \Rightarrow t^{T} \cdots\right\rangle \\
t^{T_{0} \times T_{1}} & ::=\left(t^{T_{0}}, t^{T_{1}}\right) \\
t^{\mathbb{P} T} & ::=\left\{z^{T} \mid P\right\} \\
t^{\mathbb{B}} & ::=\text { True | False } \\
t^{\mathbb{N}} & ::=0|1| 2 \mid \cdots
\end{array}
$$

where $[D] \preceq T_{2}$.

For clarity of presentation we permit the meta-variable $C$ (etc.) to range over terms of power type, and $S$ (etc.) to range over sets of schema type. The latter are schemas. We let $U$ (etc.) range over operation schemas. These are schemas involving both before and after observations. We can always write the type of such operation schemas as $\mathbb{P}\left(T^{\text {in }} \curlyvee T^{\text {out }}\right.$ ) where $T^{\text {in }}$ is the type of the input sub-binding and $T^{\text {out }} t^{\prime}$ is the type of the output sub-binding. We also permit binding concatenation, written $t_{0} \star t_{1}$ when the alphabets of $t_{0}$ and $t_{1}$ are disjoint. This is, in fact, exclusively used for partitioning bindings in operation schemas into before and after components, so the terms involved are necessarily disjoint. We lift this operation to sets (of appropriate type):

$$
C_{0} \star C_{1}=_{d f}\left\{z_{0} \star z_{1} \mid z_{0} \in C_{0} \wedge z_{1} \in C_{1}\right\}
$$

The same restriction obviously applies here: the types of the sets involved must be disjoint.

We employ the notation b.P and b.t (generalising binding selection) which is adapted from [i. Suppose that $\left\{\mathbf{z}_{0} \cdots \mathbf{z}_{n}\right\}$ is the alphabet set of $t$, then $t . P$ is $P\left[\mathbf{z}_{0} / t \cdot \mathbf{z}_{0}\right] \cdots\left[\mathbf{z}_{n} / t \cdot \mathbf{z}_{n}\right]$.

The formulæ of $\mathcal{Z}_{\mathcal{C}}$ delineate a typed bounded predicate logic.

$$
P::=\text { false }\left|t^{T}=t^{T}\right| t^{T} \in C^{\mathbb{P} T}|\neg P| P \vee P \mid \exists z^{T} \in C^{\mathbb{P} T} \bullet P
$$

The logic of $\mathcal{Z}_{\mathcal{C}}$ is classical, so the remaining logical operations are available by definition. We also, as usual, abbreviate $\neg\left(t^{T} \in C^{\mathbb{P} T}\right)$ to $t^{T} \notin C^{\mathbb{P} T}$.

A crucial observation is unicity of types: every term and set of $\mathcal{Z}_{\mathcal{C}}$ has a unique type. We can make great use of this observation. It enables us to remove type decoration in most circumstances.

\section{A.1.2 The logic of $\mathcal{Z}_{\mathcal{C}}$}

The judgements of the logic have the form $\Gamma \vdash P$ where $\Gamma$ is a set of formulæ. 
312 An analysis of total correctness refinement models for partial relation semantics I

The logic is presented as a natural deduction system in sequent form. We shall omit all data (entailment symbol, contexts, type etc.) which remain unchanged by a rule. In the rule $\left(\exists^{-}\right)$, the variable $y$ may not occur in $C, P_{0}, P_{1}$ nor any other assumption.

$$
\begin{aligned}
& \frac{P_{0}}{P_{0} \vee P_{1}}\left(\vee_{\mathrm{o}}^{+}\right) \quad \frac{P_{1}}{P_{0} \vee P_{1}}\left(\vee_{1}^{+}\right) \quad \frac{P_{0} \vee P_{1} \quad P_{0} \vdash P_{2} \quad P_{1} \vdash P_{2}}{P_{2}}\left(\vee^{-}\right) \\
& \frac{P \vdash \text { false }}{\neg P}\left(\neg^{+}\right) \quad \frac{P \quad \neg P}{\text { false }}\left(\text { false }^{+}\right) \quad \frac{\neg \neg P}{P}\left(\neg^{-}\right) \quad \frac{\text { false }}{P}\left(\text { false }^{-}\right) \\
& \frac{P[z / t] \quad t \in C}{\exists z \in C \bullet P}\left(\exists^{+}\right) \quad \frac{\exists z \in C \bullet P_{0} \quad y \in C, P_{0}[z / y] \vdash P_{1}}{P_{1}}\left(\exists^{-}\right) \\
& \overline{\Gamma, P \vdash P}(\text { ass }) \quad \overline{t=t}(\text { ref }) \quad \frac{t=t^{\prime} \quad P[z / t]}{P\left[z / t^{\prime}\right]}(\text { sub }) \\
& \overline{\downarrow \cdots \mathbf{z}_{i} \Rightarrow t_{i} \cdots \nabla \cdot \mathbf{z}_{i}=t_{i}}\left(\Rightarrow_{0}^{=}\right) \quad \overline{\downarrow \cdots \mathbf{z}_{i} \Rightarrow t \cdot \mathbf{z}_{i} \cdots \nabla=t^{\left[\cdots \mathbf{z}_{i} \in T_{i} \cdots\right]}}\left(\Rightarrow_{1}^{=}\right) \\
& \overline{\left(t_{0}, t_{1}\right) \cdot 1=t_{0}}\left(()_{\mathrm{o}}^{\overline{=}}\right) \quad \overline{\left(t_{0}, t_{1}\right) \cdot 2=t_{1}}\left(()_{1}^{=}\right) \quad \overline{(t .1, t .2)=t}\left(()_{2}^{\bar{y}}\right) \\
& \frac{P[z / t]}{t \in\{z \mid P\}}\left(\{\}^{+}\right) \quad \frac{t \in\{z \mid P\}}{P[z / t]}\left(\{\}^{-}\right) \\
& \frac{t_{0} \equiv t_{1}}{t_{0}=t_{1}}(e x t) \quad \frac{t^{T} \cdot \mathbf{z}_{i}=t_{i} \quad\left[\cdots \mathbf{z}_{i}: T_{i} \cdots\right] \preceq T}{\left(t\left\lceil\left[\cdots \mathbf{z}_{i} \in T_{i} \cdots\right]\right) \cdot \mathbf{z}_{i}=t_{i}\right.}(\uparrow=)
\end{aligned}
$$

where

$$
t_{0} \equiv t_{1}=_{d f} \forall z \in t_{0} \bullet z \in t_{1} \wedge \forall z \in t_{1} \bullet z \in t_{0}
$$

The transitivity of equality and numerous equality congruence rules for the various term forming operations are all derivable in view of rule $(s u b)$. In particular, we can prove that set-equality in $\mathcal{Z}_{\mathcal{C}}$ is extensional.

The following weakening rule is admissible and is incorporated within the system.

$$
\frac{\Gamma \vdash P_{1}}{\Gamma, P_{0} \vdash P_{1}}(w k)
$$

The carrier sets (with the expected derived rules) are easily defined this system:

$$
T=\left\{z^{T} \mid \text { True }\right\}
$$

The ambiguity presents no problem, since only types appear as superscripts and only sets appear after the membership relation.

\section{A.1.3 Restricted equality}

In many contexts we need to compare bindings over a common restricted type.

Definition A.1 Let $T \preceq T_{0}$ and $T \preceq T_{1}$.

$$
t_{0}^{T_{0}}={ }_{T} t_{1}^{T_{1}}={ }_{d f} t_{0}\left\lceil T=t_{1} \uparrow T\right.
$$

A restricted form of membership is very useful for establishing the schema calculus. In this case the types are uniquely determined by the syntax and do not appear in the notation.

Definition A.2 Let $T_{0} \preceq T_{1}$.

$$
t^{T_{1}} \dot{\epsilon} C^{\mathbb{P} T_{0}}={ }_{d f} t \uparrow T_{0} \in C
$$




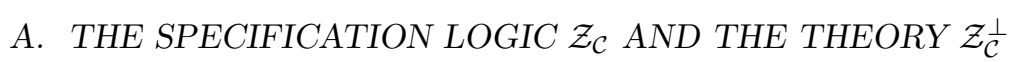

\section{A.1.4 Functions}

We will need total functions over types. These are easily introduced.

\section{Definition A.3}

$$
T_{0} \rightarrow T_{1}=_{d f}\left\{g \in \mathbb{P}\left(T_{0} \star T_{1}\right) \mid \operatorname{unicity}(g) \wedge \operatorname{total}(g)\right\}
$$

Note that functions are modelled as subsets of $T_{0} \star T_{1}$ rather than $T_{0} \times T_{1}$, and that for notational convenience, we let $g$ (etc.) range over terms of type $\mathbb{P}\left(T_{0} \star T_{1}\right)$. In fact we only do this when such a term is a function.

When $g$ is known to be an element of $T_{0} \rightarrow T_{1}$ and $z_{0} \in T_{0}$, we will write $g z_{0}$ (as usual) for the unique element $z_{1} \in T_{1}$ such that $z_{0} \star z_{1} \in g$.

\section{A.2 $\mathcal{Z}_{\mathcal{C}}^{\perp}$}

In $\mathcal{Z}_{\mathcal{C}}^{\perp}$ we introduce new constants (to play the rôle of "undefined" values) at every type. Thus we postulate new constants $\perp^{T}$ in every type $T$. There are, additionally, a number of axioms which ensure that all the new $\perp^{T}$ values interact properly.

$$
\begin{gathered}
\overline{\left\langle\mathbf{z}_{0} \Rightarrow \perp{ }^{T_{0}} \cdots \mathbf{z}_{n} \Rightarrow \perp^{T_{n}}\right\rangle=\perp\left[\mathbf{z}_{0}: T_{0} \cdots \mathbf{z}_{n}: T_{n}\right]} \\
\overline{\left(\perp^{T_{0}}, \perp^{T_{1}}\right)=\perp^{T_{0} \times T_{1}}} \\
\overline{\left\{z^{T} \mid z=\perp^{T}\right\}=\perp^{\mathbb{P} T}}
\end{gathered}
$$

For example:

$$
\perp^{\left[\mathbf{z}_{0}: T_{0} \cdots \mathbf{z}_{n}: T_{n}\right]} \cdot \mathbf{z}_{i}=\perp^{T_{i}}(0 \leq i \leq n)
$$

Note that these are the only axioms concerning undefined terms, hence, the term forming constructions are non-strict with respect to the $\perp^{T}$ values.

In order to facilitate the proper interpretation of schemas we will need to introduce the natural carrier sets which are those sets of elements of types which explicitly exclude the $\perp^{T}$ values:

Definition A.4 The natural carriers for each type are defined by closing:

$$
\mathbb{N}={ }_{d f}\left\{z^{\mathbb{N}} \mid z \neq \perp^{\mathbb{N}}\right\}
$$

and:

$$
\mathbb{B}={ }_{d f}\left\{z^{\mathbb{B}} \mid z \neq \perp^{\mathbb{B}}\right\}
$$

under the operations of cartesian product, powerset and schema set.

In $\mathcal{Z}_{\mathcal{C}}$ the schema calculus is introduced by means of definitions such as:

$$
[S \mid P]=_{d f}\left\{z^{T} \mid z \in S \wedge z \cdot P\right\}
$$

for atomic schemas, and:

$$
S_{0}^{\mathbb{P} T_{0}} \vee S_{1}^{\mathbb{P} T_{1}}=_{d f}\left\{z^{T_{0} \curlyvee T_{1}} \mid z \dot{\epsilon} S_{0} \vee z \dot{\epsilon} S_{1}\right\}
$$

for schema disjunction.

In interpreting the schema calculus in $\mathcal{Z}_{\mathcal{C}}^{\perp}$ we systematically modify the defintions so that the values are drawn over the natural carriers rather than the type. For example:

$$
[S \mid P]=_{d f}\{z \in T \mid z \in S \wedge z \cdot P\}
$$

defines atomic schemas, and:

$$
S_{0}^{\mathbb{P} T_{0}} \vee S_{1}^{\mathbb{P}^{T_{1}}}={ }_{d f}\left\{z \in\left(T_{0} \curlyvee T_{1}\right) \mid z \dot{\epsilon} S_{0} \vee z \dot{\epsilon} S_{1}\right\}
$$

defines schema disjunction.

As a result the schema calculus is hereditarily $\perp$-free, since obviously: 
314 An analysis of total correctness refinement models for partial relation semantics I

Proposition A.5

$$
t \in U^{T} \Rightarrow t \in T
$$

We will also need the extended carriers. These are defined for all types as follows:

Definition A.6

$$
T_{\perp}={ }_{d f} T \cup\left\{\perp^{T}\right\}
$$

\section{A.3 Conservativity}

$\mathcal{Z}_{\mathcal{C}}^{\perp}$ is conservative over $\mathcal{Z}_{\mathcal{C}}$. The presence of projection terms $(t .1, t .2, t . z)$ in $\mathcal{Z}_{\mathcal{C}}^{\perp}$ makes the definition of a simple translation of $\mathcal{Z}_{\mathcal{C}}^{\perp}$ into $\mathcal{Z}_{\mathcal{C}}$ difficult. The appropriate approach is to show that there is a translation of $\mathcal{Z}_{\mathcal{C}}^{\perp}{ }^{-}$into $\mathcal{Z}_{\mathcal{C}}$, where $\mathcal{Z}_{\mathcal{C}}^{\perp}{ }^{-}$is roughly $\mathcal{Z}_{\mathcal{C}}^{\perp}$ with the projections removed. Having shown that $\mathcal{Z}_{\mathcal{C}}^{\perp}$ is a conservative extension of $\mathcal{Z}_{\mathcal{C}}$ we can then show that $\mathcal{Z}_{\mathcal{C}}^{\perp}$ is a conservative (in fact a definitional) extension of $\mathcal{Z}_{\mathcal{C}}^{\perp^{-}}$. This then completes the task.

\section{A.3.1 The theory $\mathcal{Z}_{\mathcal{C}}^{\perp^{-}}$}

The language of $\mathcal{Z}_{\mathcal{C}}^{\perp}$ is that of $\mathcal{Z}_{\mathcal{C}}^{\perp}$ with the projection terms removed. The $\mathcal{Z}_{\mathcal{C}}^{+}$logic is the logic of $\mathcal{Z}_{\mathcal{C}}^{\perp}$ with the equality rules for the projection terms removed and the following equality rules (which are derivable in $\mathcal{Z}_{\mathcal{C}}^{\perp}$ and $\mathcal{Z}_{\mathcal{C}}$ ) added.

$$
\frac{t_{0}=t_{2} \quad t_{1}=t_{3}}{\left(t_{0}, t_{1}\right)=\left(t_{2}, t_{3}\right)} \quad \frac{\cdots t_{i}=v_{i} \cdots}{\downarrow \cdots \mathbf{z}_{i} \rightrightarrows t_{i} \cdots \downarrow=\downarrow \cdots \mathbf{z}_{i} \Rightarrow v_{i} \cdots \downarrow}
$$

\section{A.3.2 $\mathcal{Z}_{\mathcal{C}}^{\perp^{-}}$is conservative over $\mathcal{Z}_{\mathcal{C}}$}

Definition A.7 We define a mapping from the language of $\mathcal{Z}_{\mathcal{C}}^{\perp}{ }^{-}$into that of $\mathcal{Z}_{\mathcal{C}}$. We omit the type superscripts which can easily be inferred.
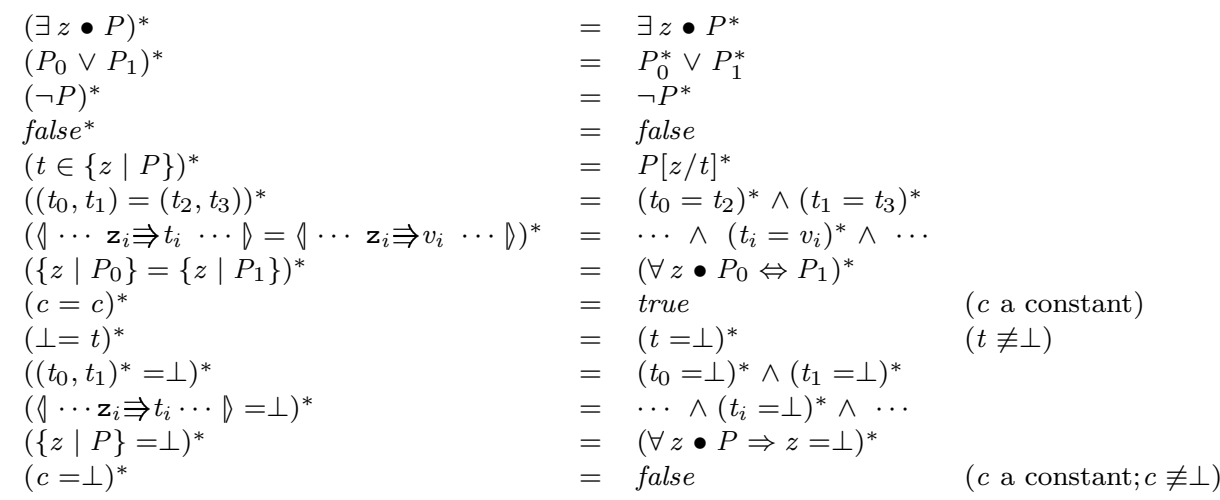

Note that if $P$ is in the language of $\mathcal{Z}_{\mathcal{C}}$ then $P^{*}=P$.

Proposition A.8 If $\Gamma \vdash_{\mathcal{Z}_{\mathcal{C}}^{\perp-}} P$ then $\Gamma^{*} \vdash_{\mathcal{Z}_{\mathcal{C}}} P^{*}$

Proof. By induction on the structure of derivations. Since the translation is a direct structural recursion for the most part, the majority of the cases are immediate. We illustrate with one of the new axioms. Consider:

$$
\overline{\left(\perp^{T_{0}}, \perp^{T_{1}}\right)=\perp^{T_{0} \times T_{1}}}
$$

Note simply that $\left(\left(\perp^{T_{0}}, \perp^{T_{1}}\right)=\perp^{T_{0} \times T_{1}}\right)^{*}=\left(\perp^{T_{0}}=\perp^{T_{0}}\right)^{*} \wedge\left(\perp^{T_{1}}=\perp^{T_{1}}\right)^{*}=$ true $\wedge$ true $=$ true.

Corollary A.9 $\mathcal{Z}_{\mathcal{C}}^{\perp^{-}}$is conservative over $\mathcal{Z}_{\mathcal{C}}$. 
A. TTHE SPECIFICATION LOGICZ

A.3.3 $\mathcal{Z}_{\mathcal{C}}^{\perp}$ is conservative over $\mathcal{Z}_{\mathcal{C}}^{\perp^{-}}$

In fact $\mathcal{Z}_{\mathcal{C}}^{\perp}$ is a definitional extension of $\mathcal{Z}_{\mathcal{C}}^{\perp}$.

Definition A.10 For all types, $T_{0}$ and $T_{1}$ we define:

$$
\mathbf{1}^{\mathbb{P}\left(\left(T_{0} \times T_{1}\right) \times T_{0}\right)}={ }_{d f}\left\{\left(\left(z_{0}, z_{1}\right), z_{2}\right)^{\left(T_{0} \times T_{1}\right) \times T_{0}} \mid z_{0}=z_{2}\right\}
$$

Note, that 1 satisfies totality and unicity so $1 \in\left(T_{0} \times T_{1}\right) \rightarrow T_{0}$

Then we can define the first projection operator of $\mathcal{Z}_{\mathcal{C}}^{\perp}$ (and of course, of $\mathcal{Z}_{\mathcal{C}}$ ).

Definition A.11

$$
t^{T_{0} \times T_{1}} \cdot 1=\mathbf{1}^{\mathbb{P}\left(\left(T_{0} \times T_{1}\right) \times T_{0}\right)}(t)
$$

Similar definitions can be established for the second projection and for binding projections. With these in place one easily derives the missing $\mathcal{Z}_{\mathcal{C}}^{\perp}$ equality rules for projections.

Proposition A.12 $\mathcal{Z}_{\mathcal{C}}^{\perp}$ is conservative over $\mathcal{Z}_{\mathcal{C}}^{\perp^{-}}$

Received 18th January 2002, revised 10 February 2003. 